\title{
ESR investigation of NR and IR rubber vulcanized with different cross-linking agents
}

\author{
P. Posadas ${ }^{1 *}$, M. A. Malmierca ${ }^{1}$, A. González-Jiménez ${ }^{1}$, L. Ibarra $^{1}$, A. Rodríguez ${ }^{1}$, \\ J. L. Valentin ${ }^{1}$, T. Nagaoka ${ }^{2}$, H. Yajima ${ }^{2}$, S. Toki ${ }^{3}$, J. Che ${ }^{4}$, L. Rong ${ }^{4}$, B. S. Hsiao ${ }^{4}$ \\ ${ }^{1}$ Instituto de Ciencia y Tecnología de Polímeros (CSIC) C/ Juan de la Cierva, 3. 28006 Madrid, Spain \\ ${ }^{2}$ Department of Chemistry, Tokyo University of Science, Shinjuku-ku, 162-9601 Tokyo, Japan \\ ${ }^{3}$ National Metal and Materials Technology Center, Mahidol university, Salaya campus, 73170 Nakhon Pathm, Thailand \\ ${ }^{4}$ Department of Chemistry, State University of New York, Stony Brook, 11794-3400 New York, USA
}

Received 25 May 2015; accepted in revised form 9 August 2015

\begin{abstract}
This study evaluates the formation of radical species in natural rubber (NR) and poly-isoprene rubber (IR) during the vulcanization process and the uniaxial deformation of the formed networks by means of Electron Spin Resonance (ESR). Vulcanization of NR and IR always shows a radical pathway, where the different vulcanization systems dictate the concentration of radical species in the course of this complex process. The greatest concentration of radicals were detected during the vulcanization with sulfur/accelerator based on efficient systems (EV), followed by conventional (CV) and sulfur donor systems, whereas azide and organic peroxide agents showed smaller concentration of radicals. Independently of the vulcanization system, certain amount of radicals was detected on the vulcanized samples after the end of the vulcanization process. Comparison between different matrices demonstrates that NR always shows higher concentration of radicals than IR in the vulcanization process as well as during uniaxial deformation, fact that could be associated to the presence of nonrubber components in NR.
\end{abstract}

Keywords: rubber, vulcanization, electron spin resonance

\section{Introduction}

Technical application of rubbers requires the vulcanization process to create a three dimensional polymer network which provides the characteristic elastic properties of these materials. Depending on the vulcanization system and the type of rubber, in addition to vulcanization conditions, the chemistry of this process changes, having important consequences on the network structure and consequently, on the elastic properties of these rubber materials. The most common vulcanization agents on rubber technology are sulfur and organic peroxides. In the case of sulfur systems, the discussion concerning the nature of the reactions involved in the vulcanization process, i.e. ionic or free radical pathways, is still ongoing [1-4]. Several works conclude that radical mechanisms are required to justify the crosslink structures formed on NR after the vulcanization with sulfur [2-6]: sulfur cross-links of different lengths, mainly polysulfidic bonds, pendant groups, cyclic sulfides and cis/trans isomerization of the double bonds and/or conjugated sequences in the polymer backbone [7]. In addition, the low reaction efficiency and the long time required to complete vulcanization process do not recommend the use of sulfur without accelerators for commercial purposes [6]. The sulfur-accelerator systems reduce the optimum vulcanization time, improve the elastic properties of NR and minimize the side-reactions during the vulcanization process. They are classi-

\footnotetext{
${ }^{*}$ Corresponding author, e-mail: pposadas@ictp.csic.es

(C) BME-PT
} 
fied as conventional (CV), semi-efficient (semi$\mathrm{EV}$ ) and efficient (EV) systems depending on the accelerator/sulfur ratio (usually in a range between 0.2 and 12, for the extreme cases). The NR samples usually show different and characteristic mechanical and viscoelastic properties, fatigue and ageing resistance according to the sulfur/accelerator system employed in the vulcanization process $[1,7]$. On the other hand, vulcanization of NR with organic peroxides provides materials with higher concentration of non-elastic network defects and rubber network structures characterized by the formation of $\mathrm{C}-\mathrm{C}$ cross-links with more heterogeneous spatial distribution, as compared to the samples vulcanized with sulfur-accelerators [6]. These structural differences have a critical impact on the drop of elastic properties of these materials, being caused by the complex free radical mechanism (with different radical pathways and side reactions) that characterizes the vulcanization of NR with organic peroxides. Nevertheless, peroxide crosslinking has also several advantages in comparison with sulfur cure with regard to different applications of the rubbers (e.g., compression set at elevated temperatures, covulcanization, etc.) [8]. According to this statement, the main objective of this work is to determine the presence and concentration of free radicals on the vulcanization process of NR and poly-isoprene rubber concerning the different vulcanization systems and their influence on the network structure and physical properties of NR. In this sense, Electron Spin Resonance (ESR) is a powerful and unique tool for detecting radicals. ESR has been previously used to evaluate the radical concentration on polyolefins and different rubbers [9-12] vulcanized with organic peroxides. The application to other vulcanization systems, e.g. sulfur based systems, has been limited to model compounds or the analysis of the vulcanization system without rubber [1]. Recently, ESR has been applied to study the polybutadiene vulcanization mechanism [13].

In addition to the study of vulcanization process, the second part of this work will be focused on the study of radicals generated during uniaxial deformation of NR [14], fact that could be related with variations in the network structure and consequently in the final properties of NR after cycles of deformation.

\section{Experimental section}

\subsection{Materials, preparation of samples and rheological measurements}

The studied compounds were based on standardized natural rubber (SMRCV60), kindly supplied by Malaysian Rubber, Kuala Lumpur, Malaysia and poly cis-1,4-polyisoprene (IR) kindly supplied by Kraton Polymers, Amsterdam, The Netherlands $\left(\right.$ KRATON $^{\circledR}$ IR307). Five different vulcanization systems were used in this work: two sulfur based systems with different accelerator/sulfur ratios (A/S) which correspond to a conventional system (named $\mathrm{CV}$ with $\mathrm{A} / \mathrm{S}=0.2$ ) and an efficient system (named $\mathrm{EV}$ with $\mathrm{A} / \mathrm{S}=0.2$ ), respectively; a sulfur donor system (DPTT), an organic peroxide (DCP) and a diazide molecule (BSA) as it was reported in Table 1.

For the ESR measurements during the vulcanization process, samples were prepared in toluene solutions

Table 1. Recipes of the samples

\begin{tabular}{|c|c|c|c|c|c|c|c|c|c|c|}
\hline & NR-DPTT & NR-CV & NR-EV & NR-BSA & NR-DCP & IR-DPTT & IR-CV & IR-EV & IR-BSA & IR-DCP \\
\hline NR & \multicolumn{5}{|c|}{100} & \multicolumn{5}{|c|}{ - } \\
\hline IR & \multicolumn{5}{|c|}{ - } & \multicolumn{5}{|c|}{100} \\
\hline Zinc oxide* & 5 & 5 & 5 & - & - & 5 & 5 & 5 & - & - \\
\hline Stearic acid ${ }^{*}$ & 1 & 1 & 1 & - & - & 1 & 1 & 1 & - & - \\
\hline DPTT $^{a)}$ & 5 & - & - & - & - & 5 & - & - & - & - \\
\hline Sulfur ${ }^{*}$ & - & 2.5 & 0.56 & - & - & - & 2.5 & 0.56 & - & - \\
\hline $\mathrm{CBS}^{\mathrm{b})}$ & - & 0.5 & 6.72 & - & - & - & 0.5 & 6.72 & - & - \\
\hline $\mathrm{A} / \mathrm{S}^{\mathrm{c})}$ & - & 0.2 & 12 & - & - & - & 0.2 & 12 & - & - \\
\hline $\mathrm{BSA}^{\mathrm{d})}$ & - & - & - & 3.13 & - & - & - & - & 3.13 & - \\
\hline $\mathrm{DCP}^{\mathrm{e})}$ & - & - & - & - & 1.4 & - & - & - & - & 1.4 \\
\hline
\end{tabular}

a) Dipentamethylene thiuram tetrasulfide

b) N-cyclohexyl-2-bezonthiazole sulfenamide

c)Accelerator/sulfur rate

d) Benzene-1,3-disulphonylazide prepared by reacting 1,3 benzene dicloro-sulfonyl with sodium azide in our laboratory [15]

e) Bis (1-methyl-1-phenylethyl) peroxide (Dicumyl peroxide, (DCP), 97\% purity) which was supplied by Merck KGaA (Darmstadt, Germany) and it was used as received

${ }^{*}$ Sulfur, zinc oxide and stearic acid were supplied by Sigma-Aldrich. 
(5\% in volume) according the recipes summarized in Table 1. Finally rubber films with thickness about $0.5 \mathrm{~mm}$ were obtained by casting. Rubber films were dried at room temperature until constant weight.

For other measurements, the rubber compounding was executed on a laboratory two-roll mill with a cylinder diameter of $15 \mathrm{~cm}$, a length of $30 \mathrm{~cm}$ and a friction ratio between them of $1: 1.15$, following the habitual protocol employed in rubber science and technology. Rolls were kept at $40-50^{\circ} \mathrm{C}$ during the mixing procedure.

Vulcanization of rubber samples was executed using a hydraulic press heated by electric resistances at $150^{\circ} \mathrm{C}$ at their respective optimum vulcanization time $\left(t_{97}\right)$, whereas the samples vulcanized with azides were vulcanized at $105^{\circ} \mathrm{C}$ at their corresponding $t_{97}$.

Vulcanization time was determined by the torquetime curve obtained from the Monsanto Moving Die Rheometer, model MDR 2000E. These vulcanization curves were used to obtain the optimum vulcanization time, $t_{97}$, defined as the time required for reaching the $97 \%$ of the maximum elastic torque.

\subsection{Determination of cross-link density}

Cross-link densities were determined by equilibrium swelling measurements. Equilibrium swelling experiments in toluene were performed according to the protocol described elsewhere [16]. The solvent density $\rho_{\mathrm{s}}=0.87 \mathrm{~g} / \mathrm{mL}$ was used for toluene.

The influence of insoluble components (such as zinc oxide) was corrected according to Equation (1) [16]:

$$
V_{\text {rubber }}=\frac{w_{\mathrm{d}}}{\rho_{\text {rubber }}}-\left[w_{\mathrm{d}} \cdot\left(\frac{w_{\mathrm{ZnO}}}{w_{\text {compound }}}\right) \cdot \frac{1}{\rho_{\mathrm{ZnO}}}\right]
$$

where $w_{\mathrm{d}}$ is the sample weight after drying, $\rho_{\text {rubber }}$ is the density of NR $\left(0.92 \mathrm{~g} / \mathrm{cm}^{3}\right) ; w_{\mathrm{ZnO}}$ is the $\mathrm{ZnO}$ weight, $w_{\text {compound }}$ is the recipe weight and $\rho_{\mathrm{ZnO}}$ is the density of $\mathrm{ZnO}\left(5.6 \mathrm{~g} / \mathrm{cm}^{3}\right)$.

Assuming an affine model to characterize the elastic behavior of swollen networks, cross-link density (v) was obtained by using the Flory-Rehner equation (Equation (2)) [17]:

$\nu\left[\frac{\mathrm{mole}}{\mathrm{cm}^{3}}\right]=\frac{-\left[\ln \left(1-V_{\text {rubber }}\right)+V_{\text {rubber }}+\chi V_{\text {rubber }}^{2}\right]}{V_{1}\left[V_{\text {rubber }}-\frac{V_{\text {rubber }}}{2}\right]}$

where $V_{\text {rubber }}$ is the volume fraction of polymer in the swollen mass, $V_{1}$ is the molar volume of the solvent and $\chi$ is the Flory-Huggins polymer-solvent interaction parameter.

It is well known that the $\chi$ parameter depends on the solvent-rubber pair and the volumetric fraction of rubber. For this reason a variable $\chi$ parameter has been used according to the recommendations made elsewhere [16].

\subsection{Electron spin resonance (ESR) experiments}

Electron spin resonance (ESR) measurements were performed on a JEOL spectrometer ES-FA200 Xband. The external standard of ESR spectrum for $g$ value and line width were the third and fourth resonant lines of $\mathrm{Mn}^{2+}$, which were at 2.034 and 1.981, respectively. The sweep time was 30 seconds. Two accumulations were enough to get an ESR absorption curve with high signal to noise ratio. A double integration of the first derivative curve of ESR signal at room temperature from benzene solution of 4-hidroxy-2,2,6,6,-tetramethyl piperidinooxyl radical (TEMPOL) with a known radical concentration was used as a standard to calculate the radical concentration of rubber samples [14, 18-20]. The radical concentration is defined per gram of rubber.

Measurements on un-vulcanized rubber films in order to evaluate the formation of radicals during the vulcanization process were performed at $150^{\circ} \mathrm{C}$, except azide sample where the temperature was $105^{\circ} \mathrm{C}$. We decided the starting time as fast as the sample is set in the chamber since the temperature of the sample reach the setting temperature easily due to the thinness of the sample $(0.5 \mathrm{~mm})$ and measuring time (1 minute), although the data in initial one minute has some uncertainty necessarily.

Measurements of vulcanized rubbers under deformation were carried out by a home-built stretching machine combined with the ESR instrument at room temperature. Samples were stretched under a constant stretching speed of $10 \mathrm{~mm} / \mathrm{min}$ up to a desired strain while ESR measurements were taken. The strain was increased up to $200 \%$ which was the maximum strain due to an instrumental limitation. Details for measurements were described elsewhere [21].

\section{Results}

\subsection{Formation and evolution of free radicals during the vulcanization process}

The vulcanization process for IR and NR was monitored by their corresponding rheometer curves as 
shown in Figure 1. Both rubber matrices show similar trends in the vulcanization curves for the different systems.

The DPTT system shows the fastest cross-linking reaction and together with the EV system are characterized by higher increment of torque (associated to the formation of cross-links between the rubber chains, as it is shown in Table 2) as compared with the other vulcanization systems. Although both systems show some reversion in the vulcanization curve, i.e. decrease in the torque after reaching the maximum value, the sample vulcanized with CV system presents the highest reversion. This process has been related with rubber chain scission and the breaking down of cross-links during the vulcanization process. In the samples vulcanized with low $\mathrm{A} / \mathrm{S}$ ratio this phenomenon is more evident because of the thermolability of polysulfidic bonds which are predominant for CV systems [22].

Opposite to sulfur/accelerator systems, the vulcanization with peroxide (DCP) does not show any scorch time, because the cross-linking reaction takes place immediately after the fast homolytic decomposition of DCP at the vulcanization temperature. In a similar way, the scorch time for DPTT system is very short and it is not recorded by the rheometer [23]. The main goal of this investigation is to evaluate the formation of free radicals during the vulcanization process according to the different vulcanization systems. For this reason, ESR experiments were performed on uncured compounds at the same temperature as the rheometer curves, e.g. $150^{\circ} \mathrm{C}$ for all samples with exception of samples vulcanized with BSA which were measured at $105^{\circ} \mathrm{C}$. For that reason the results extracted from the rheometer could be used to understand the ESR measurements.

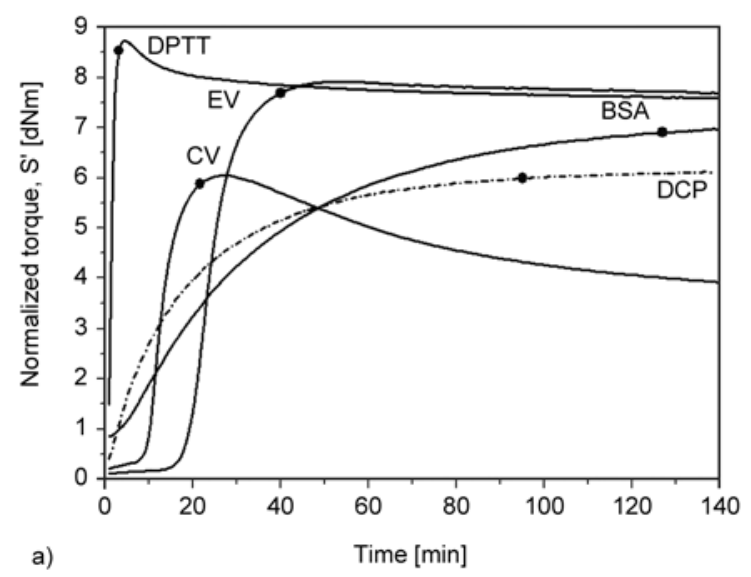

\subsubsection{Peroxide system}

Figure 2 shows the first derivative of the ESR spectra for IR and NR samples vulcanized with DCP at different vulcanization times. The graph shows the magnetic field range from 318 to $326 \mathrm{H} / \mathrm{mT}$.

Independently of the vulcanization time, it is possible to identify four peaks for the IR sample (Figure $2 \mathrm{a}$ ), in a similar way as in the case of polyolefins crosslinked with DCP [10].

The mechanism of peroxide vulcanization is clearly based on free radical pathways. It is initiated by the thermal homolytic scission of the peroxide molecule, creating two radicals. These radicals are able to react with diene polymer backbones (e.g. IR and NR) via abstraction of the more labile allylic hydrogen atoms or via addition to the double bonds, forming polymer radicals. In the simplest cross-linking reaction (ignoring numerous other side reactions that do not generate junctions between rubber chains), two competing termination reactions may take place $[8,24-26]$. On one hand, recombination of two polymer radicals to form a tetra-functional carbon-carbon cross-link and on the other hand, addition of one polymer radical to a double bond of another rubber

Table 2. densities of the samples vulcanized at their optimum vulcanization times measured by swelling experiments

\begin{tabular}{|c|c|c|c|}
\hline Samples NR & $\begin{array}{c}\text { Crosslink } \\
\text { density } \\
{\left[\mathrm{mol}^{\prime} / \mathrm{cm}^{3} \cdot 10^{5}\right]}\end{array}$ & Samples IR & $\begin{array}{c}\text { Crosslink } \\
\text { density } \\
{\left[\mathrm{mol}^{2} / \mathrm{cm}^{3} \cdot 10^{5}\right]}\end{array}$ \\
\hline DPTT & $10.41 \pm 0.03$ & DPTT & $10.3 \pm 0.2$ \\
\hline $\mathrm{CV}$ & $6.64 \pm 0.03$ & $\mathrm{CV}$ & $6.24 \pm 0.03$ \\
\hline $\mathrm{EV}$ & $8.84 \pm 0.02$ & EV & $8.4 \pm 0.2$ \\
\hline BSA & $6.8 \pm 0.3$ & BSA & $7.2 \pm 0.3$ \\
\hline DCP & $5.49 \pm 0.09$ & DCP & $6.3 \pm 0.2$ \\
\hline
\end{tabular}

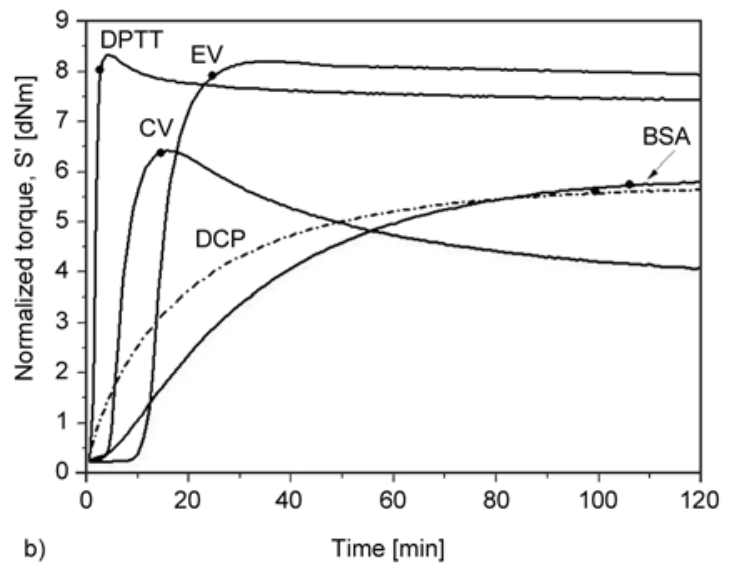

Figure 1. Rheometric curves a) IR samples. b) NR samples. Symbols $(\bullet)$ indicate the optimum vulcanization time $\left(t_{97}\right)$ for each sample. 

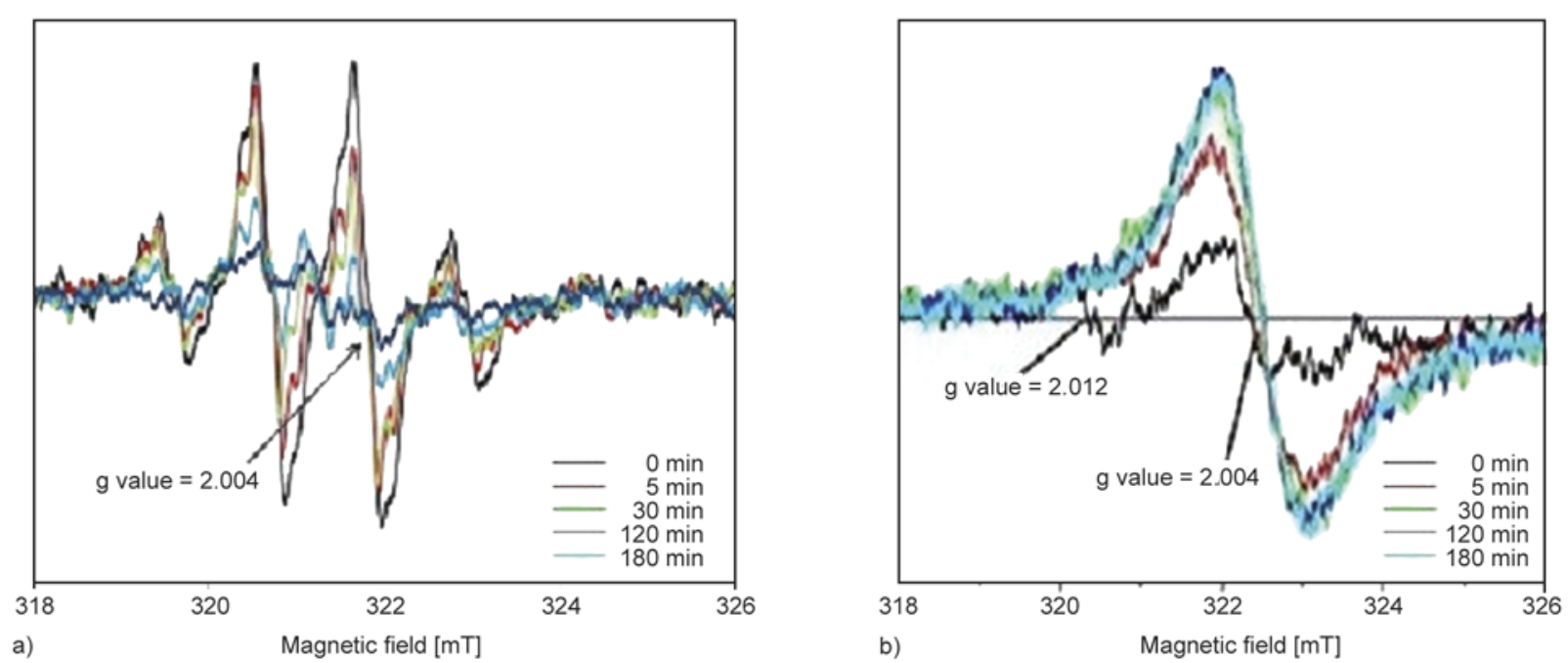

Figure 2. The first derivative of ESR spectra of DCP samples at different time during vulcanization at $150^{\circ} \mathrm{C}$, a) IR samples and b) NR samples

chain giving rise to a cross-link and a new active free radical.

Taking into account this mechanism, the process is initiated by the thermal homolytic scission of the peroxide. Nevertheless, these radicals are not detected by the ESR experiments because they are very reactive radicals with a very short life time at the measured temperatures [27-30]. In addition, it is important to take into consideration the unavoidable uncertainties associated to the ESR data acquired during the first minute.

The intensity of the signal in the ESR spectrum depends on the stability of radicals (which determines the rate of decay) rather than the rate of their formation. For that reason, signals observed in the ESR spectra for IR samples vulcanized with DCP (Figure 2a) should be related to the formation of different polymer radicals. According to the intensity ratio and the $g$ values around 2.0048 it is possible to assign them to allylic radicals. When the vulcanization time increases, the intensity of this signal increases because more allylic radicals are generated but some of them react with another polymer radical creating carbon-carbon crosslinks. After 120 minutes, the intensity of this signal diminishes because most of the allylic radicals have reacted. This statement agrees with the vulcanization curve (obtained with the rheometer) and determines the end of the vulcanization process.

It is important to remark that the ESR signal becomes broader with the vulcanization time because the formation of additional cross-links restricts the mobility of the radicals.
Previous work based on the study of free radicals in cross-linked EPDM rubbers attributed the peak with the $g$ value around 2.012 to alkyl radicals generated in the initial steps of the peroxide vulcanization process. These alkyl radical intermediates are quite reactive and will readily react via abstraction of the allylic hydrogen in the diene monomer [11]. In the case of NR or IR rubber, the concentration of double bonds are higher than EPDM rubber and for that reason, alkyl radicals are less probable to be detected. Nevertheless, the NR ESR spectrum recorded at the earliest stage seems to show a signal with a $g$ value close to 2.012 (Figure 2b).

In the case of NR sample vulcanized with DCP, the allyl radicals (assigned to the signal at $g=2.004$ ) increases with the vulcanization time and become broader because the presence of non-rubber components which could hinder the mobility of the radicals. In addition these non-rubber components are able to reduce the ESR spectral resolution due to the incomplete averaging of anisotropies of magnetic parameters [11].

\subsubsection{Sulfur cure systems}

The sulfur vulcanization has been studied by using three different systems: conventional (CV with high sulfur concentration, $\mathrm{A} / \mathrm{S}=0.2$ ), efficient ( $\mathrm{EV}$ with high accelerator concentration, $\mathrm{A} / \mathrm{S}=12$ ) and a sulfur donor (DPTT without addition of sulfur).

In spite of sulfur vulcanization mechanism is still a controversial issue, there is a widespread agreement about the basic steps of this process, which can be divided into three stages $[2,3]$ : 
1. Accelerators chemistry where the active sulfurating agent is created.

2. Cross-link chemistry where the cross-links between rubber chains are formed.

3. Post-vulcanization chemistry where the shortening of sulfur bridges in addition to other degradation process takes place.

According to this mechanism, sulfur vulcanization is a complex process where radical route is considered as one of the main pathways which could explain the network structures reported for NR [2-5].

In this sense, the evaluation of model compounds with solid-state ${ }^{13} \mathrm{C}-\mathrm{NMR}$ reveals that the main structures generated during sulfur vulcanization correspond to allylic attacks in both the secondary and primary carbons [31-37].

\section{CV system}

Figure 3 shows the first derivative of ESR spectra for IR-CV and NR-CV samples at different vulcanization times. This sulfur/accelerator system seems to form a more complex mixture of free radicals than the peroxide system according to the multiple peaks observed in Figure 3. This behavior could be associated on one hand to the number of ingredients (chemical species) in the recipe that are able to generate radicals and, on the other hand, the numerous reactions between them.

At the beginning of the vulcanization process (chemistry of accelerators) the concentration of free radicals increases very fast (see Figure 3 ) and it is possible to identify many different species $(g=1.974$, $1.978,2.004$ and 2.024, respectively) which could be formed by the reaction of sulfur, zinc oxide,

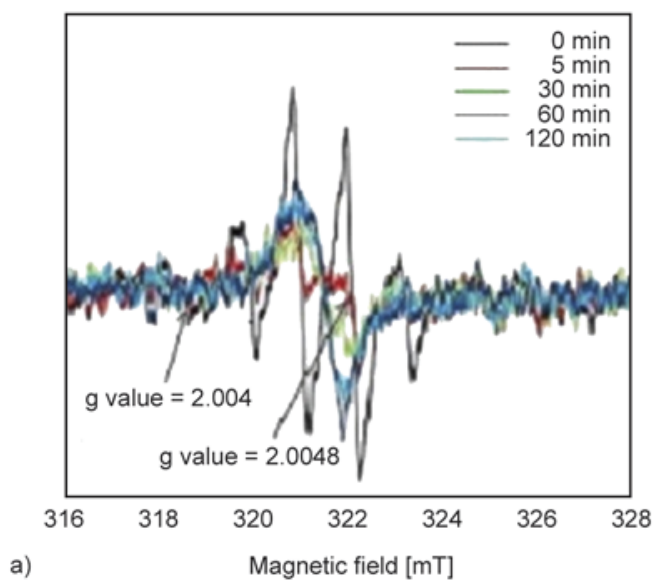

stearic acid and accelerator system [2]. The active sulfurating agent formed during this step reacts with the rubber chain and it is able to graft the sulfur atoms in the macromolecule. This precursor moiety is converted into a cross-link by the direct reaction with another rubber molecule via a persulfenyl intermediate $\left(\mathrm{RS}_{\mathrm{y}}{ }^{\bullet}\right)$ [2]. For that reason, during the cross-linking reaction, most of the free radical species (evolved in the accelerator chemistry) disappear, but the total concentration of radicals in the system seems to be unaffected.

In order to understand this fact, it is mandatory to take into consideration that once the sulfur cross-links are formed, further desulfuration or degradation reactions take place (according to the vulcanization curves showed in Figure 1). In the desulfuration reactions, the removal of sulfur atoms changes the length distribution of sulfur bridges without any variation in the cross-link density [2]. In contrast, the degradation reactions lead to loss of cross-links and it results in complex structures like cyclic sulfides, main chain modifications and cis/trans isomerization [2]. Rubber samples vulcanized with conventional systems are characterized by the predominance of polysulfidic linkages in the network structure $\left(\mathrm{C}-\mathrm{S}_{\mathrm{x}}-\mathrm{C}\right.$, where $x$ identify the number of sulfur atoms in the sulfidic bridge), which are more sensitive to temperature than shorter di- or mono-sulfidic cross-links because of their lower dissociation energy (approximately $150 \mathrm{~kJ} / \mathrm{mol}$ for polysulfidic bridges with four sulfur atoms) [2]. After the dissociation process, another active sulfur radical species are formed and they could react again to i) reform other sulfur cross-links with different lengths and/or

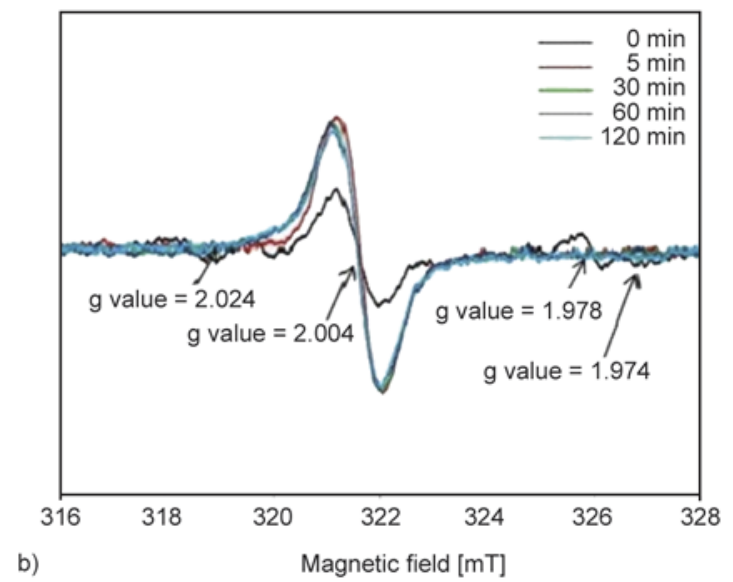

Figure 3. The first derivative of ESR spectra of CV samples at different time during vulcanization at $150^{\circ} \mathrm{C}$, a) IR samples and b) NR samples 
ii) form side products like $\mathrm{RS}_{\mathrm{Z}} \mathrm{H}$ [2]. For that reason, the free radical species identified in the last steps of the vulcanization process correspond to carbon and sulfur radicals in rubber materials situated at $g=$ 2.004 (these radicals are not distinguished because they have almost the same $g$ value).

\section{EV-cure system}

Figure 4 shows the first derivative of ESR spectra for IR and NR samples vulcanized with an efficient vulcanization system at different reaction times. Spectra split into four peaks at short time, which can be attributed to radical species formed during the accelerator chemistry, as it was explained for the CV system.

The $g$-values in the ESR derivatives around 2.004 correspond to carbon or sulfur radicals generated in the course of the cross-linking reaction or during

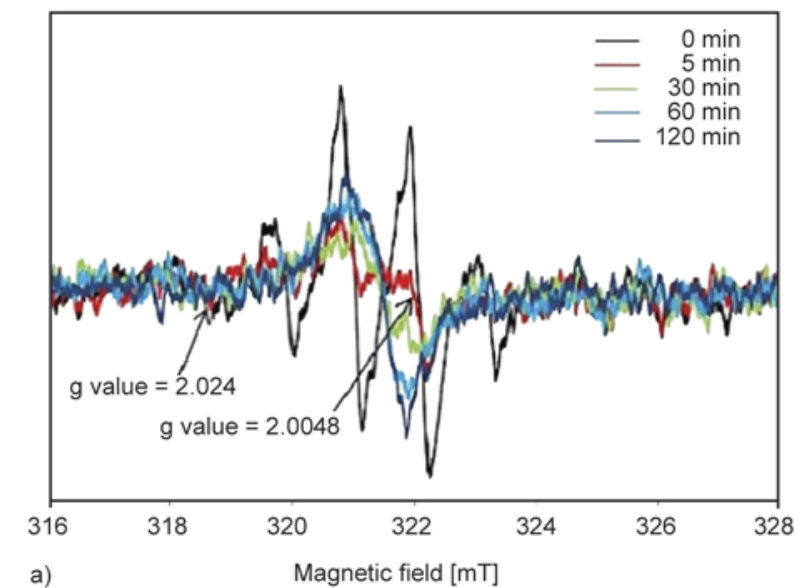

the post vulcanization stage according to the statements widely explained for the CV systems.

\section{DPTT system}

Figure 5 shows the first derivatives of ESR spectra for samples vulcanized with the sulfur donor DPTT at different times. These spectra show the same peaks than the other sulfur-based systems (CV and EV) at the initial vulcanization time. However, in the samples vulcanized with DPTT, these radicals remain active throughout the vulcanization process.

It is important to remind that DPTT is capable to generate sulfidic cross-links without the addition of sulfur to the rubber compound. In the case of sulfur donor systems, the thermal scission of DPTT takes place via homolytic cleavage producing free radical species. Although other unsymmetrical cleavage is also possible, these reactions are less probable

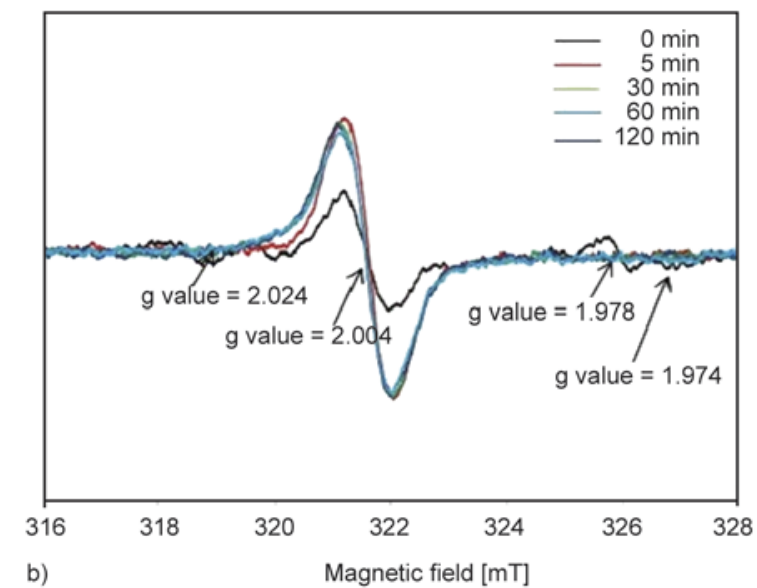

Figure 4. The first derivative of ESR spectra of EV samples at different time during vulcanization at $150^{\circ} \mathrm{C}$, a) IR samples and b) NR samples
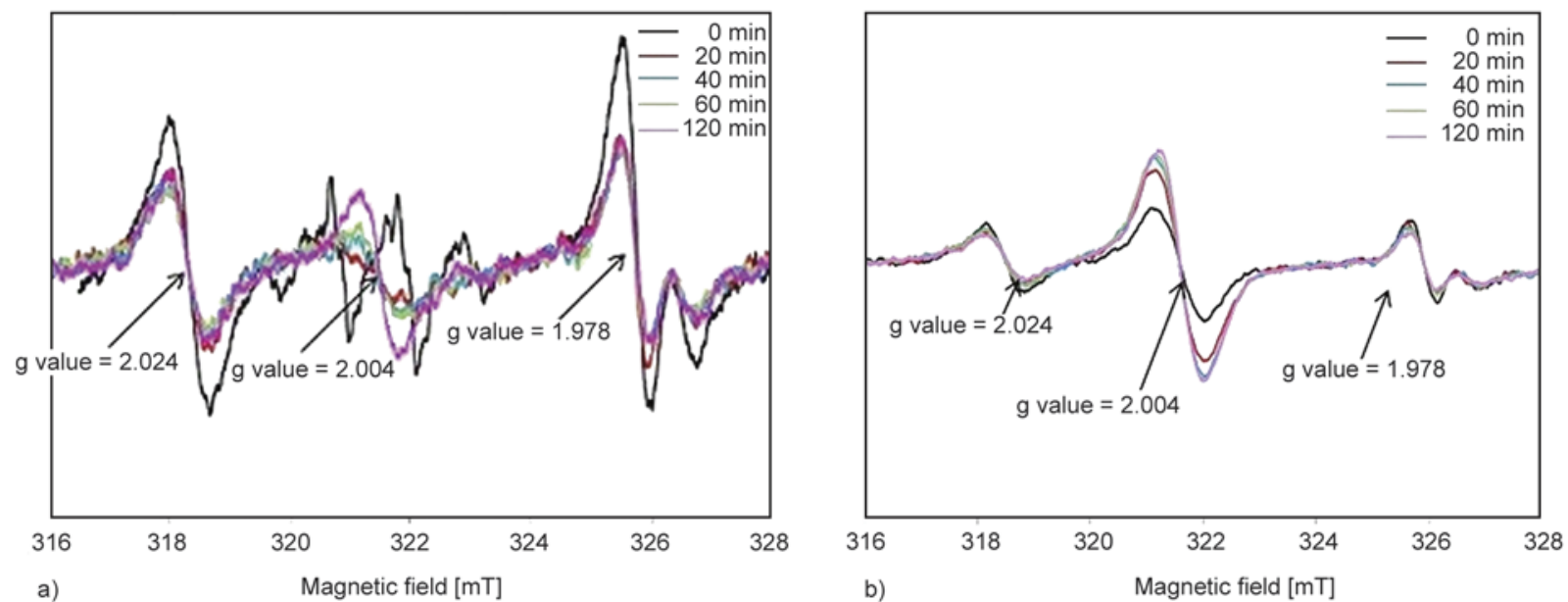

Figure 5. The first derivative of ESR spectra of DPTT samples at different time during vulcanization at $150^{\circ} \mathrm{C}$, a) IR samples and b) NR samples 
because require the breakdown of the more stable $\mathrm{C}-\mathrm{S}$ bond. For this reason, only the two central sulfur atoms of DPTT molecule are able to create crosslinks [22, 23].

According this statement, the detection of ESR signals corresponding to the radical species formed during the chemistry of accelerators at larger vulcanization times identifies that their lifetime is enlarged in the DPTT system in comparison with the other sulfur based vulcanization systems. This fact demonstrates that these radicals are more stable in the sulfur donor system, because there are not free sulfur molecules ready to react with them.

\subsubsection{BSA system}

Figure 6 shows the first derivatives of ESR spectra for samples vulcanized with BSA at different vulcanization times.

Besides sulfur and peroxide systems, there are some other less common vulcanization agents such as organic azides, which are capable to cross-link unsaturated rubber as NR, BR, SBR, EPDM and PDMS $[15,38,39]$. At temperatures near $100^{\circ} \mathrm{C}$, azides react by 1,3 dipolar cycle-addition mechanism giving rise to hetero-cycles of the 1,3 triazoline type. These derivates decompose releasing nitrogen and giving rise to aziridine and imine derivatives, the amount obtained from these products vary, depending on the type of olefin involved [15]. However, sulfonyl azides decompose to form a sulfonyl nitrene at elevated temperatures (above $155^{\circ} \mathrm{C}$ ). The nitrene exist in both the singlet and the triplet state. Both states can react with hydrocarbons but they do it in different ways. The reaction involving the singlet nitrene

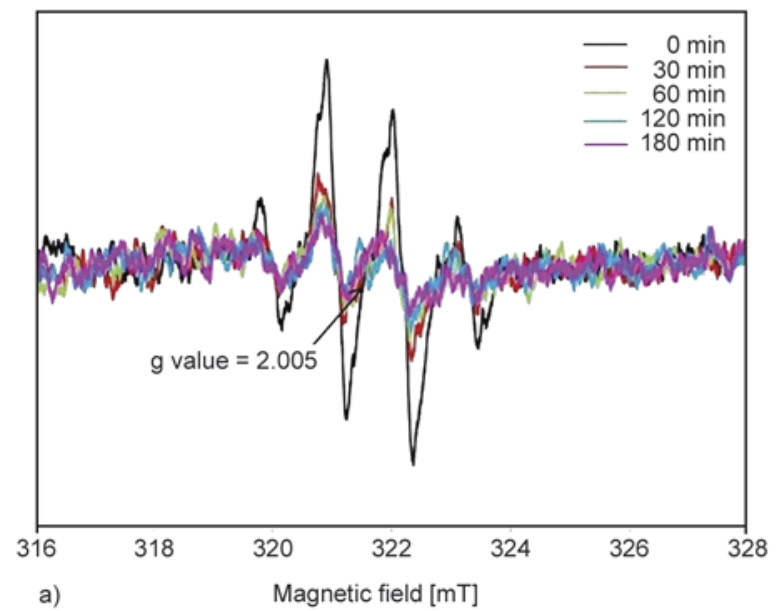

is an insertion at the $\mathrm{C}-\mathrm{H}$ bond giving a secondary sulfonamide, while the triplet nitrene reacts by abstraction of a proton from the hydrocarbon resulting in the formation of a macroradical and this reaction can follow by a recombination of two macroradicals to form a cross-link [40].

The ESR measurements of BSA system were executed at $105^{\circ} \mathrm{C}$ in order to promote the cross-linking reaction in absence of radicals, according to the mechanism describe before. At this temperature, the cross-linking mechanism of azide molecules is controlled through the formation of the heterocyclic $1,2,3$, triazoline intermediate, which is unstable at higher temperatures. Nevertheless, BSA system is able to create nitrogenous radical which seems to have a similar behavior than alkoxy radicals obtained during the vulcanization with organic peroxides, as it is shown in Figure 6.

\subsection{Comparison of radical amounts produced by different vulcanization system}

The concentration of radical species produced by the different vulcanization agents are compared in Figure 7. The first important conclusion extracted from this study is that all the vulcanization systems, e.g. organic peroxide, sulfur/accelerator, sulfur donor system and azide molecules, produce free radical species during the vulcanization process of NR and IR matrices. The appearance of ESR-detectable species requires the accumulation of high concentration of radicals. Usually carbon-centered radicals are extremely reactive and consequently not detectable by ESR at the vulcanization temperature. However, the formation of cross-links reduces the

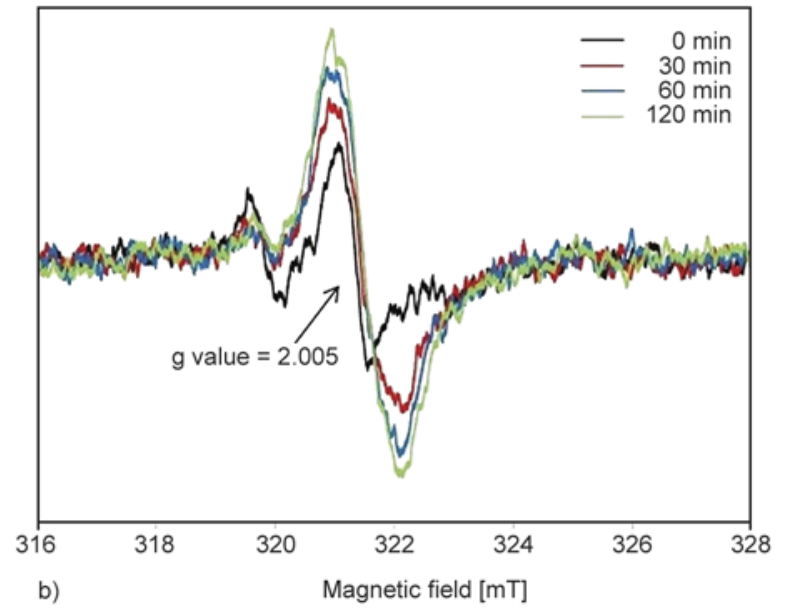

b) Magnetic field [mT]

Figure 6. The first derivative of ESR spectra of BSA samples at different time during vulcanization at $105^{\circ} \mathrm{C}$, a) IR samples and b) NR samples 

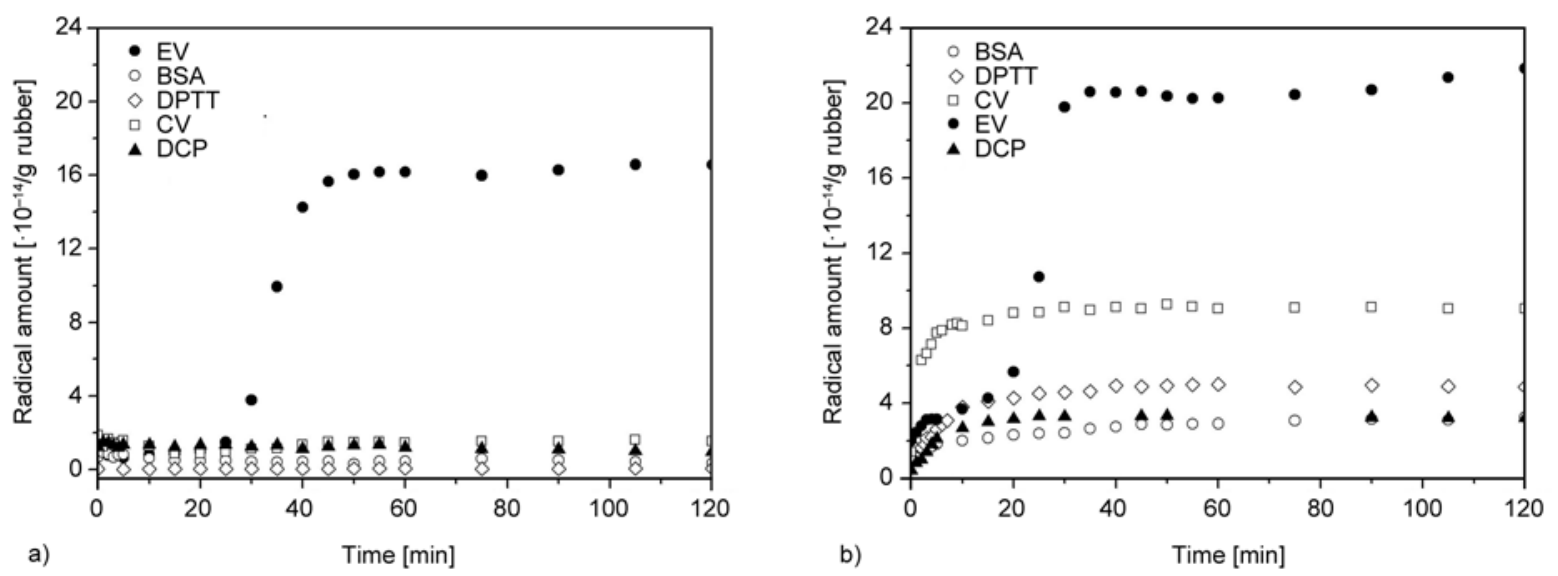

Figure 7. Radical concentration produced by different vulcanization agent a) in IR rubber and b) in NR rubber

mobility and the accessible space to rubber segments, limiting the reaction of radicals and enlarging their lifetime.

In a previous work, the peroxide vulcanization of different ethylene-propylene-diene rubbers were investigated by using ESR technique, showing the increase of ESR intensities with the vulcanization time until it reach a maximum, followed by a long decay of this intensity at larger times because of the consumption of the formed radicals [11].

Opposite to this behavior, NR and IR matrices show an increasing concentration of radical species with the vulcanization time until a plateau is reached. The concentration of radicals remain almost constant over the optimum vulcanization time (as measured by rheometric curves), indicating that the radicals generated during vulcanization process have a long lifetime. The enhanced stability of radicals in NR matrix is caused by the high concentration of double bonds in the polymer backbone in comparison with the EPDM matrices [11]. In addition, the more restricted mobility of polymer radicals with the vulcanization time (because of the development of cross-linking reaction) not only enlarge the lifetime of radicals, but also it worsens the ESR spectral resolution due to the incomplete averaging of anisotropies of magnetic parameters [11].

In this sense, the ESR spectra for NR samples always show worst resolution and higher radical concentration than the IR samples, independently of the vulcanization system. Differences between rubber matrices are explained neither by the differences in the chemical structure of polymer radicals nor by the effect of cross-links in the segmental mobility of the polymer backbone (both NR and IR have almost the same cross-link density at the optimum vulcaniza- tion time according the equilibrium swelling experiments). These results seem to indicate the importance of non-rubber components in NR during the creation of different radical species during the vulcanization process, independently of the used cure system. Additionally, thermal degradation of these non-rubber components at the vulcanization temperature would be one of the main factors in the formation of radicals, although they have not a clear influence on the creation of additional cross-links.

The EV system always shows the highest concentration of free radicals (almost double) during the vulcanization. In order to understand this result, the two closer sulfur/accelerator systems, e.g. EV and $\mathrm{CV}$, have been compared. In first term, and taking into account the previous statements, the higher concentration of free radicals during the vulcanization with EV system should be mainly ascribed to the highest amount of accelerant (CBS) in the recipe. According to previous studies, the molecule of CBS produces radicals at $150{ }^{\circ} \mathrm{C}[14,21]$.

The cross-link density is around 30\% higher for samples vulcanized with the EV system. This fact could enhance the lifetime of radical species, but it is not enough to justify the difference in the concentration of radicals. In second term, the thermal stability of mono- and di-sulfidic linkages - which are the main type of cross-links in the EV systems - is higher in comparison to the poly-sulfidic bridges that are mainly formed during the vulcanization with the CV system. The shorter sulfidic cross-links are characterized by higher dissociation energies $(289 \mathrm{~kJ} / \mathrm{mol}$ for di-sulfidic bonds [2]). This difference in bond association energies is related to the formation of monosulfidic radicals $\mathrm{RS}^{\bullet}$, which are not stabilized by neighboring sulfur atoms [2]. This fact 
should reduce the radical species for EV systems during the post-vulcanization stage.

\subsection{Evolution of radical amounts under the tensile deformation}

Spectra for all samples were made at different strain. All resonance lines show a broad absorption with the same $g$ value of 2.004, which is assigned to carbon and sulfur radicals as it was described in the previous sections. Since the sample volume in the ESR cavity decreases with strain, the raw spectra were corrected by the volume change assuming that Poisson's ratio of the samples was kept as 0.5 . The experiments were rather difficult because the thin stripe samples were elongated in a small ESR cavity, samples were easily torn during elongation. Only four samples were succeeded to get the data. As an example, radical concentrations for sulfur accelerated vulcanization at different strains are showed in Figure 8.

During deformation, the rupture of network points and polymer chains may occur accompanying the formation of radicals. Network densities for all the studied samples are similar (see Table 2), consequently the differences in the creation of radicals during deformation should be directly related to the different nature of network points formed during the vulcanization process.

The radical concentration remains constant with strain in the vulcanized IR samples as it is showed in Figure 8a. Only the sample vulcanized with the EV system shows a slight increase in the radical concentration at low strain. Opposite to this behavior, the radical concentration increases with strain for the NR samples, independently of the vulcanization system. In addition, it is important to point out the cer-

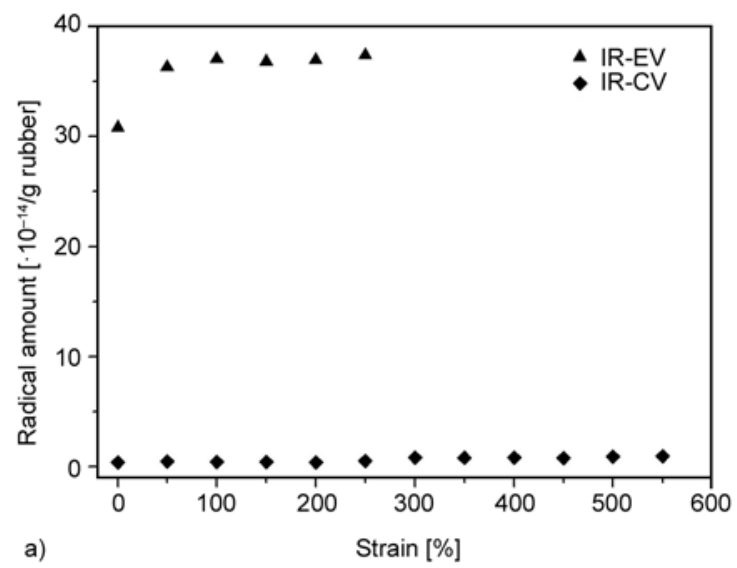

tain amounts of radicals are detectable by ESR in the un-stretched state (strain 0 ) for samples vulcanized with EV system, demonstrating their stability and extremely long lifetime.

\section{Conclusions}

ESR detectable species requires the accumulation of high concentration of radicals. For that reason, the extremely reactive carbon-centered radicals are not detectable by ESR at the vulcanization temperature. However, the formation of cross-links reduces the mobility and accessible conformations of rubber segments, limiting the reaction of radical species and enlarging their lifetime. In this sense, the first important conclusion is that all the studied vulcanization systems, e.g. organic peroxide, sulfur/accelerator, sulfur donor system and azide molecules, produce free radical species during the vulcanization process of NR and IR matrices.

In the case of peroxide and BSA systems only the radical with the $g$ value around 2.004 is detected by ESR. This radical is attributed to the allylic radical in the polymer backbone generated during the crosslinking reaction. Sulfur systems seem to form more complex mixture of free radicals according to the higher number of peaks detected by ESR. This behavior could be associated on one hand, to the high number of ingredients inside the rubber matrix that are able to generate radicals and on the other hand, the numerous reactions between them. At the beginning of the vulcanization process, the concentration of free radicals increases very fast and it is possible to identify many different kinds of radicals species $(g=1.974,1.978,2.004$ and 2.024) which could be formed by the reaction of sulfur, zinc oxide, stearic acid and the accelerator system. These radicals dis-

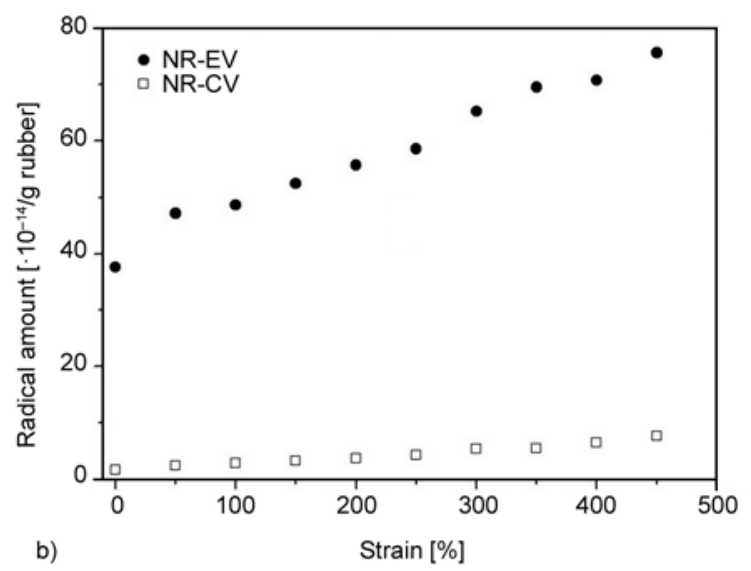

Figure 8. Evolution of radical amount vs. strain in a) IR E and IR-CV systems, b) in NR-E and NR-CV systems 
appear during the cross-linking reaction in the case of sulfur/accelerator systems. However, these radicals remain active for longer times in those samples vulcanized with sulfur donor system. The higher lifetime of these radicals could be attributed to their enhanced stability as a consequence of the lack of reactive sulfur molecules in the system.

The ESR signals for NR samples are always broader than in the case of IR samples due to the presence of non-rubber components which could hinder the mobility of radicals and additionally they provoke the incomplete averaging of anisotropies of magnetic parameters.

In addition, the concentration of radicals remains almost constant over the optimum vulcanization time measured by the rheometer, indicating that radicals generated during the vulcanization process have a long lifetime. EV vulcanization system shows the highest concentration of radical species, because of large amount of the accelerator (CBS). During deformation, the NR samples always show higher concentration of radicals than the IR counterparts. Additionally, the concentration of radical species increases with deformation for NR compounds.

\section{Acknowledgements}

The work was partially supported by the Spanish Ministry of Science and Innovation (MICINN) under project MAT-201123476. M.A.M. would also like to express their gratitude to Consejo Superior de Investigaciones Cientifícas (CSIC) for her Jae-Pre-088 fellowships. A. G.-J. acknowledges MEC for a FPU fellowship. JLV thanks to Ministerio de Ciencia e Innovación (Spain) for his Ramon y Cajal contract.

H.Y. and T.N. are indebted to Prof. Dr. Masayoshi Ito of Tokyo University of Science for his adequate advice about ESR measurements.

S.T., J.C., L.R., and B.S.H. acknowledge the financial support from the National Science Foundation (DMR-0906512).

\section{References}

[1] Coleman M. M., Shelton J. R., Koenig J. L.: Sulfur vulcanization of hydrocarbon diene elastomers. Industrial and Engineering Chemistry Product Research and Development, 13, 154-166 (1974). DOI: $10.1021 / \mathrm{i} 360051 \mathrm{a} 002$

[2] Ghosh P., Katare S., Patkar P., Caruthers J. M., Venkatasubramanian V.: Sulfur vulcanization of natural rubber for benzothiazole accelerated formulations: From reaction mechanisms to a rational kinetic model. Rubber Chemistry and Technology, 76, 592-693 (2003). DOI: $10.5254 / 1.3547762$
[3] Heideman G., Datta R. N., Noordermeer J. W. M., van Baarle B.: Activators in accelerated sulfur vulcanization. Rubber Chemistry and Technology, 77, 512-541 (2004). DOI: $10.5254 / 1.3547834$

[4] Krejsa M. R., Koenig J. L.: A review of sulfur crosslinking fundamentals for accelerated and unaccelerated vulcanization. Rubber Chemistry and Technology, 66, 376-410 (1993). DOI: $10.5254 / 1.3538317$

[5] Manik S. P., Barnerjee S.: Sulfenamide accelerated sulfur vulcanization of natural rubber in presence and absence of dicumyl peroxide. Rubber Chemistry and Technology, 43, 1311-1326 (1970).

DOI: $10.5254 / 1.3547331$

[6] Valentín J. L., Posadas P., Fernández-Torres A., Malmierca M. A., González L., Chassé W., Saalwächter K.: Inhomogeneities and chain dynamics in diene rubbers vulcanized with different cure systems. Macromolecules, 43, 4210-4222 (2010). DOI: $10.1021 / \mathrm{ma} 1003437$

[7] Chapman A. V., Porter M.: Natural rubber science and technology. Oxfor Science Publications, New York (1988).

[8] Dluzneski P. R.: Peroxide vulcanization of elastomers. Rubber Chemistry and Technology, 74, 451-492 (2001). DOI: 10.5254/1.3547647

[9] Camara S., Gilbert B. C., Meier R. J., van Duin M., Whitwood A. C.: EPR studies of peroxide decomposition, radical formation and reactions relevant to crosslinking and grafting in polyolefins. Polymer, 47, 46834693 (2006). DOI: $10.1016 /$ j.polymer.2006.04.015

[10] Liu J., Yu W., Zhao C., Zhou C.: EPR/rheometric studies on radical kinetics in melt polyolefin elastomer initiated by dicumyl peroxides. Polymer, 48, 2882-2891 (2007). DOI: 10.1016/j.polymer.2007.03.053

[11] Zachary M., Camara S., Whitwood A. C., Gilbert B. C., van Duin M., Meier R. J., Chechik V.: EPR study of persistent free radicals in cross-linked EPDM rubbers. European Polymer Journal, 44, 2099-2107 (2008). DOI: 10.1016/j.eurpolymj.2008.04.008

[12] Yamazaki T., Seguchi T.: Electron spin resonance study on chemical crosslinking reaction mechanisms of polyethylene using a chemical agent. V. Comparison with polypropylene and ethylene-propylene copolymer. Journal of Polymer Science Part A: Polymer Chemistry, 38, 3383-3389 (2000).

DOI: $10.1002 / 1099-0518(20000915) 38: 18<3383:: A I D-$ POLA170>3.0.CO;2-M

[13] Dondi D., Buttafava A., Zeffiro A., Palamini C., Lostritto A., Giannini L., Faucitano A.: The mechanisms of the sulfur-only and catalytic vulcanization of polybutadiene: An EPR and DFT study. European Polymer Journal, 62, 222-235 (2015). DOI: $10.1016 /$ j.eurpolymj.2014.11.012 
[14] Toki S., Takagi R., Ito M., Hsiao H. S.: Rupture, orientation and strain-induced crystallization of polymer chain and network in vulcanized polyisoprene during uniaxial deformation by in-situ electron spin resonance (ESR) and synchrotron X-ray analysis. Polymer, 52, 2453-2459 (2011).

DOI: $10.1016 /$ j.polymer.2011.03.037

[15] de Benito G. J. L., Ibarra R. L. M., González H. L.: The use of benzene 1,3-sulphonyl azide as an elastomer cross-linking agent. I, Dienic elastomers. Kautschuk und Gummi, Kunststoffe, 43, 146-149 (1990).

[16] Valentín J. L., Carretero-Gonzalez J., Mora-Barrantes I., Chassé W., Saalwächter K.: Uncertainties in the determination of cross-link density by equilibrium swelling experiments in natural rubber. Macromolecules, 41, 4717-4729 (2008).

DOI: $10.1021 / \mathrm{ma} 8005087$

[17] Flory P., Rehner J.: Statistical mechanics of crosslinked polymer networks II. Swelling. Journal of Chemical Physics, 11, 521-526 (1943).

DOI: $10.1063 / 1.1723792$

[18] Ito M., Isago H., Suzuki N.: ESR study of silica-filled SBR with different rubber/filler interactions. Journal of Applied Polymer Science, 108, 1385-1392 (2008). DOI: 10.1002 /app. 27843

[19] Ito M., Takizawa A.: Difference in the stability of network between natural rubber and polyisoprene vulcanizates. Kautschuk und Gummi Kunststoffe, 65, 24-27 (2012).

[20] Suzuki N., Ito M., Yatsuyanagi F.: Effects of rubber/ filler interactions on deformation behavior of silica filled SBR systems. Polymer, 46, 193-201 (2005). DOI: $10.1016 /$ j.polymer.2004.10.066

[21] Funatsu J., Takagi R., Ito M.: ESR study of vulcanizates under the deformed state. Journal of Applied Polymer Science, 113, 2791-2795 (2009).

DOI: $10.1002 /$ app.30340

[22] González L., Rodríguez A., Valentín J. L., Marcos-Fernández, A., Posadas P.: Conventional and efficient crosslinking of natural rubber: Effect of heterogeneities on the physical properties. Kautschuk und Gummi Kunststoffe, 58, 638-643 (2005).

[23] Posadas P., Fernández-Torres A., Valentín J. L., Rodríguez A., González L.: Effect of the temperature on the kinetic of natural rubber vulcanization with the sulfur donor agent dipentamethylene thiuram tetrasulphide. Journal of Applied Polymer Science, 115, 692-701 (2010).

DOI: $10.1002 / a p p .30828$

[24] González L., Rodríguez A., Marcos-Fernández A., Chamorro C.: New aspects of the crosslinking reaction of natural rubber with dicumil peroxide filler effect. Kautschuk und Gummi Kunststoffe, 47, 715-721 (1994).
[25] González L., Rodríguez A., Marcos A., Chamorro C.: Crosslink reaction mechanisms of diene rubber with dicumyl peroxide. Rubber Chemistry and Technology, 69, 203-214 (1996).

DOI: $10.5254 / 1.3538365$

[26] Valentín J. L. Fernández-Torres A., Posadas P., MarcosFernández A., Rodríguez A., González L.: Measurements of freezing-point depression to evaluate rubber network structure. Crosslinking of natural rubber with dicumyl peroxide. Journal of Polymer Science Part B: Polymer Physics, 45, 544-556 (2007).

DOI: $10.1002 /$ polb. 21060

[27] Gilbert B. C., Dobbs A. J.: Organic peroxide. Wiley, New York (1972).

[28] Shida T.: Electron spin resonance and optical studies of tert-butyl peroxide ions produced by $\gamma$-irradiation. Journal of Physical Chemistry, 72, 723-727 (1968). DOI: $10.1021 / \mathrm{j} 100848 \mathrm{a} 055$

[29] Zhou W., Zhu S.: ESR study of peroxide-induced crosslinking of high density polyethylene. Macromolecules, 31, 4335-4341 (1998). DOI: $10.1021 / \mathrm{ma970973s}$

[30] Zwolenik J. J.: Photolytic generation and kinetic electron spin resonance spectrometry of cumylperoxy radicals. Journal of Physical Chemistry, 71, 2464-2469 (1967).

DOI: $10.1021 / \mathrm{j} 100867 \mathrm{a} 012$

[31] Andreis M., Liu J., Koenig J. L.: Solid-state carbon-13 NMR studies of vulcanized elastomers. V. Observation of new structures in sulfur-vulcanized natural rubber. Journal of Polymer Science Part B: Polymer Physics, 27, 1389-1404 (1989). DOI: $10.1002 /$ polb.1989.090270702

[32] Clough R. S., Koenig J. L.: Solid-state carbon-13 NMR studies of vulcanized elastomers. VII. Sulfur-vulcanized cis-1,4 polybutadiene at 75.5 MHz. Rubber Chemistry and Technology, 62, 908-927 (1989).

DOI: $10.5254 / 1.3536283$

[33] Komoroski R. A., Shockcor J. P., Gregg E. C., Savoca J. L.: Characterization of elastomers and rubber chemicals by modern NMR techniques. Rubber Chemistry and Technology, 59, 328-346 (1986).

DOI: $10.5254 / 1.3538204$

[34] Krejsa M. R., Koenig J. L.: Solid-state C-13 NMR studies of vulcanized elastomers X. $N$ - $t$-butyl-2-benzothiazole sulfenamide accelerated sulfur vulcanization of cis-polyisoprene at $75.5 \mathrm{MHz}$. Rubber Chemistry and Technology, 65, 427-443 (1992). DOI: $10.5254 / 1.3538622$

[35] Skinner T. D.: The CBS-accelerated sulfuration of natural rubber and cis-1,4-polybutadiene. Rubber Chemistry and Technology, 45, 182-192 (1972). DOI: $10.5254 / 1.3544698$

[36] Zaper A. M., Koenig J. L.: Solid state carbon-13 NMR studies of vulcanized elastomers. II, Sulfur vulcanization of natural rubber. Rubber Chemistry and Technology, 60, 252-277 (1987).

DOI: $10.5254 / 1.3536129$ 
[37] Zaper A. M., Koenig J. L.: Solid state carbon-13 NMR studies of vulcanized elastomers. III, Accelerated sulfur vulcanization of natural rubber. Rubber Chemistry and Technology, 60, 278-297 (1987).

DOI: $10.5254 / 1.3536130$

[38] Damiron D., Okhay N., Al Akhrass S., Cassagnau P., Drockenmuller E.: Crosslinked PDMS elastomers and coatings from the thermal curing of vinyl-functionalized PDMS and a diazide aliphatic crosslinker. Journal of Polymer Science Part A: Polymer Chemistry, 50, 98-107 (2012).

DOI: $10.1002 /$ pola.24991
[39] De Benito G. J. L., Ibarra R. L. M., González H. L.: The use of 1,3 bis benzene sulphonyl azide as an elastomer crosslinking agent. II. Saturated elastomers. Kautschuk und Gummi Kunststoffe, 43, 697-700 (1990).

[40] Jørgensen J. K., Ommundsen E., Stori A., Redford K.: Synthesis, characterisation and decomposition of 1,3benzene disulfonyl azide; A cross-linking agent for polyolefins. Polymer, 46, 12073-12080 (2005).

DOI: $10.1016 /$ j.polymer.2005.10.077 